\title{
Pays de Galles et développement durable : « un modèle pour d'autres régions et pays»?
}

Wales and Sustainable Development: "a Model for Other Regions and Countries"?

Stéphanie Bory

\section{OpenEdition}

\section{Journals}

Édition électronique

URL : http://journals.openedition.org/rfcb/2321

DOI : $10.4000 /$ rfcb.2321

ISSN : 2429-4373

Éditeur

CRECIB - Centre de recherche et d'études en civilisation britannique

Référence électronique

Stéphanie Bory, «Pays de Galles et développement durable : « un modèle pour d'autres régions et pays»? », Revue Française de Civilisation Britannique [En ligne], XXIII-3 | 2018, mis en ligne le 01 décembre 2018, consulté le 01 mai 2019. URL : http://journals.openedition.org/rfcb/2321 ; DOI : $10.4000 /$ rfcb. 2321

Ce document a été généré automatiquement le 1 mai 2019.

\section{cc) (†) $\odot$}

Revue française de civilisation britannique est mis à disposition selon les termes de la licence Creative Commons Attribution - Pas d'Utilisation Commerciale - Pas de Modification 4.0 International. 


\title{
Pays de Galles et développement durable : « un modèle pour d'autres régions et pays»?
}

\author{
Wales and Sustainable Development: "a Model for Other Regions and \\ Countries"?
}

Stéphanie Bory

\section{Introduction}

1 Le Government of Wales Act 1998, qui a permis la mise en place d'une assemblée dévolue au pays de Galles dotée dans un premier temps de pouvoirs législatifs secondaires, a fait de la Principauté la première nation européenne à inclure le principe de développement durable dans le texte fondateur de ses institutions. L'article 121 stipule en effet: " L'Assemblée préparera un programme exposant comment elle propose, dans l'exercice de ses fonctions, de promouvoir le développement durable ${ }^{1} »$. Une annexe de la loi liste les domaines dévolus à l'Assemblée, comme l'agriculture, le tourisme, l'environnement, ou l'énergie, partiellement dévolue. Selon Phil Williams, AM (Assembly Member) pour Plaid Cymru, le parti nationaliste gallois, une telle décision devait faire du pays de Galles un modèle environnemental et lui apporter une grande visibilité :

Dans une petite nation oubliée comme le pays de Galles, nous souffrons d'un réel complexe d'infériorité et nous nous vantons souvent sans fondement que notre rôle est de diriger le monde. L'un de mes amis poètes pense que c'est dû au souvenir de Magnus Maximus qui est parti du pays de Galles et a fini empereur de Rome au nord des Alpes. Mais qui sait: si nous avons la moindre possibilité de laisser notre marque comme petite nation cela doit être en tant que nation verte et nation qui prend son engagement en faveur du développement durable très au sérieux ${ }^{2}$.

2 Pourtant, au cours de ses premières années d'existence, le bilan de l'Assemblée reste mitigé, notamment en raison des pouvoirs limités dont elle jouit. Après un second 
référendum organisé en 2011, elle obtient des pouvoirs législatifs primaires, et voit ses prérogatives élargies à nouveau par le Wales Act 2014 (notamment en matière d'énergie).

Elle obtient donc en 2011 le statut de parlement, ce qui lui a permis d'adopter deux lois majeures pour le développement durable : le Well-Being of Future Generations Act en 2015 obligeant les organismes publics à appliquer ce principe dans toute décision et politique et le Environment (Wales) Act l'année suivante - afin de promouvoir une gestion durable des ressources naturelles et réduire les émissions de gaz à effet de serre. Ces lois ont placé le pays de Galles sur le devant de la scène, ce que soulignait lors d'une conférence internationale sur le développement durable organisée à Cardiff en avril 2015 Nikhil Seth, directeur de la division pour le développement durable, le développement économique et les affaires sociales aux Nations Unies :

Le Wales Generations Act rend compte de l'esprit et de l'essence de deux décennies de travail aux Nations Unies dans le domaine du développement durable et sert de modèle pour d'autres régions et pays. [...] Nous espérons que ce que le pays de Galles accomplit aujourd'hui le monde l'accomplira demain³.

Il convient de ce fait de se demander dans quelle mesure la dévolution a permis au pays de Galles d'obtenir la visibilité à laquelle il aspire depuis longtemps et si la vision de Phil Williams est devenue réalité. Enfin, depuis la victoire, lors du référendum tenu le 23 juin 2016, du vote en faveur de la sortie de l'Union européenne, il est nécessaire de considérer si le Brexit risque de replonger la nation galloise, particulièrement présente et active à Bruxelles et dans les réseaux européens, dans l'obscurité et la marginalité.

5 Cet article se propose de présenter dans un premier temps les deux lois majeures adoptées par l'Assemblée dans le domaine environnemental, afin de dresser le bilan de son action, en étudiant notamment le gain de visibilité du pays de Galles, les conflits entre théorie et pratique, enfin la question du Brexit qui plonge les institutions galloises dans un paradoxe législatif.

\section{Deux lois majeures sur l'environnement}

6 Après le référendum de 2011 et l'obtention par l'Assemblée de pouvoirs législatifs primaires d'un seul bloc, le gouvernement dirigé par le First Minister travailliste Carwyn Jones, propose deux projets de loi majeurs sur l'environnement, adoptés en fin de législature : le Well-being of Future Generations Act et le Environment (Wales) Act.

\section{Le Well-being of Future Generations Act}

7 Cette première loi environnementale est adoptée le 29 avril 2015, après de longues discussions à l'Assemblée. Carwyn Jones annonce en juillet 2011, soit dès le début de la législature, l'intention de son gouvernement d'introduire un projet de loi sur le développement durable :

Nous allons légiférer pour intégrer le développement durable comme principe fondamental dans toutes nos actions en tant que gouvernement et dans tous les organismes publics, en introduisant un projet de loi sur le développement durable. Une telle approche distinguera le pays de Galles comme une nation attachée au développement durable, qui donne l'exemple 4

8 Cette proposition suscite de vifs débats, notamment au sujet du nom même du projet de loi. S'il est initialement prévu de nommer le projet Sustainable Development Bill, le nom est 
modifié afin de refléter davantage le nécessaire équilibre entre le bien-être des générations actuelles et futures, faisant ainsi écho à la définition Brundtland du développement durable ${ }^{5}$. Jeff Cuthbert, alors Ministre des communautés et de la lutte contre la pauvreté, indique que cette loi permettra au pays de Galles d'appliquer ce principe tel que défini à l'échelle internationale :

La Well-being of Future Generations Bill renforce les modalités existantes de gouvernance dans les organismes du secteur public cités pour l'amélioration du bien-être au pays de Galles. Le projet de loi garantit que les besoins du présent sont pourvus sans compromettre la capacité des générations futures à répondre aux leurs. Cela sera obtenu en légiférant sur le principe de développement durable ${ }^{6}$.

Un document consultatif est ensuite publié début 2012, suivi d'un second en mai et d'un livre blanc à l'automne. Un an plus tard, à l'automne 2013, un projet de loi corrigé est présenté à l'Assemblée. Une partie de ce long processus législatif est supervisé par Carl Sargeant, dont le travail et l'opiniâtreté sont loués par Anne Meikle, directrice de WWF Cymru:

Quand Carl a pris le poste, il était le quatrième Ministre à travailler sur le Well-being of Future Generations Act. Cette loi n'était ni populaire ni efficace. Carl était au départ sceptique sur la façon dont elle aiderait les gens. Mais il a écouté nos demandes d'amendements et $\mathrm{y}$ a répondu, et une fois qu'il s'est attelé à cette loi il a été très efficace pour la faire passer. Le résultat fut une loi réellement radicale pour garantir que le développement durable est au cœur des services gouvernementaux et publics, ce qui nous a apporté une reconnaissance internationale ${ }^{7}$.

Comme indiqué précédemment, elle oblige les organismes publics à appliquer le principe de développement durable dans toute décision et politique. Quarante-quatre organismes publics sont concernés, dont les autorités locales, mais aussi les National Park Authorities, les Local Health Boards, entre autres. Elle définit également sept objectifs ou «Well-Being goals »: un pays de Galles globalement responsable, prospère, flexible, plus sain, plus égalitaire, composé de communautés solidaires et possédant une culture riche et une langue dynamique.

Dans un document intitulé The Wales We Want, publié le 8 août 2016 sur le site du gouvernement, elle est décrite ainsi : «Cette loi porte sur l'amélioration du bien-être social, économique et culturel du pays de Galles ${ }^{8} »$. Elle prévoit notamment la création d'un Future Generations Commissioner for Wales, et Sophie Howe, auparavant première Deputy Police and Crime Commissioner pour le Sud du pays de Galles, est nommée à ce poste le $1^{\text {er }}$ février 2016. Elle publie très rapidement son Draft Strategic Plan 2017-2023, dans lequel elle définit de nouvelles méthodes de travail, insistant sur la nécessité d'adopter une vision à longterme, une approche intégrée et ouverte à quiconque serait intéressé par le bien-être, tout en étant collaborative et préventive. En adoptant cette législation, le pays de Galles devient la première nation à légiférer sur le développement durable.

\section{Le Environment (Wales) Act}

12 Le Well-being of Future Generations Act est couplé à un second texte, adopté un an plus tard, le 21 mars 2016, soit à quelques semaines des élections législatives galloises. Le Environment (Wales) Act 2016 comporte plusieurs volets essentiels : une gestion durable des ressources naturelles, la réduction de $80 \%$ d'ici 2050 des émissions de gaz à effet de serre par la mise en place d'un cadre législatif contraignant, la gestion des déchets et le recyclage. 

organismes publics et les entreprises privées. Selon Jessica McQuade, membre de WWF Cymru, le pays de Galles a toutes les cartes en main pour mettre en pratique le principe de développement durable :

La bonne nouvelle, c'est que nous nous trouvons à présent dans une meilleure position qu'il y a quelques années four faire face à ce défi [un pays de Galles à faible émission de carbone] grâce au Gouvernement gallois. Nous possédons une toute nouvelle loi pour aider à changer la façon dont les décisions sont prises. Le Environment Act a introduit des objectifs réglementaires pour obliger les Ministres gallois à réduire les émissions de gaz à effet de serre et fournit un programme clair d'application. Le Wellbeing of Future Generations Act impose que les considérations climatiques soient prises en compte dans toute décision prise par les organismes publics9.

Grâce à ce nouveau cadre législatif, le gouvernement annonce plusieurs mesures. Ainsi les aciéries Tata à Port Talbot (qui ont fait couler beaucoup d'encre lors des élections de mai $2016)^{10}$ vont apporter leur soutien au gouvernement pour réduire les émissions de gaz à effet de serre. De même, l'Université de Cardiff a annoncé qu'elle participerait financièrement au développement d'un outil permettant de prévoir et donc de gérer les émissions galloises pour les 30 prochaines années, et ce au nom du gouvernement, afin de lui permettre de définir des objectifs appropriés et d'adapter les budgets ${ }^{11}$. Le Docteur Monjur Mourshed, le responsable du projet au sein de l'Université, a développé un outil similaire pour le Bangladesh. Enfin, le gouvernement a débloqué une enveloppe de trois millions de livres sterling pour permettre aux autorités locales d'améliorer encore le taux de recyclage des déchets.

15 Le vote de ces deux lois environnementales majeures a donc suscité un espoir, celui de voir le pays de Galles montrer la voie, comme précédemment souligné par Nikhil Seth. Il est alors intéressant de dresser un premier bilan, à moyen terme, de ces textes, même s'il est encore tôt pour mesurer leur impact réel sur le quotidien des Gallois.

\section{Premier bilan des effets de ces deux lois majeures}

16 Afin de dresser un premier bilan des effets de ces deux lois, il convient de prendre quelques mesures phares en exemple et de se demander dans quelle mesure elles ont permis au pays de Galles de gagner en visibilité et en crédibilité, ou si, au contraire, les résultats ou l'absence de résultats n'ont fait que renforcer la marginalisation de cette nation souvent oubliée non seulement par les Britanniques, mais aussi par les Européens.

\section{Visibilité}

Le pays de Galles possède de nombreux atouts pour être extrêmement visible sur la scène environnementale internationale : il est la première nation britannique à avoir introduit une taxe sur les sacs plastique jetables ${ }^{12}$, la première nation pour la promotion du commerce équitable depuis $2008^{13}$, et la première, et à l'heure actuelle la seule, nation au monde à aider à la protection d'un coin de forêt tropicale d'une superficie équivalente à la sienne. Cette visibilité est mise en avant par Claire Raisin, directrice de Size of Wales, une organisation caritative environnementale de $\mathrm{Cardiff}^{14}$, qui ajoute à cette liste non exhaustive le Well-being of Future Generations Act, loi qu'elle considère comme avantgardiste. Le nom de cette association vient de l'expression, « Roughly the size of Wales » 
communément utilisée comme unité de mesure par les journalistes et les chercheurs pour décrire des phénomènes négatifs. Le choix d'un tel nom permet de montrer que le pays de Galles, par sa taille, peut mettre en place des politiques modèles, et ne pas seulement servir d'échelle dans des présentations alarmistes :

Le pays de Galles possède un réel potentiel pour influencer les politiques menées par d'autres pays, en mettant en avant ce qui peut être fait quand la lutte contre le réchauffement climatique est une priorité. Le pays de Galles pourrait parfaitement se faire un nom sur la scène internationale, et ce pour toutes les bonnes raisons ${ }^{15}$.

Comme souligné plus haut, l'un des volets du Well-being of Future Generations Act est la gestion des déchets et le recyclage. Deux ans après le vote de la loi, Lesley Griffiths, Ministre de l'environnement et des affaires rurales, souligne les progrès réalisés :

L'anniversaire du Well-being and Future Generations Act offre une opportunité de souligner les progrès réalisés au cours de l'année qui vient de s'écouler. Le pays de Galles continue de montrer le chemin avec une loi sur la durabilité reconnue par les Nations Unies comme 'un modèle pour d'autres régions et pays' 16 .

Elle prend comme exemple le recyclage puisque le pays de Galles occupe le troisième rang mondial pour le recyclage et est sur le point de devenir numéro un en Europe ${ }^{17}$. Les statistiques pour 2015-2016 montrent que le pays de Galles est en tête au sein du Royaume-Uni avec $60,2 \%$ de déchets recyclés, ce qui va au-delà des objectifs fixés par le gouvernement (58\%) dans Towards Zero Waste, pourcentage qui a doublé en 10 ans ${ }^{18}$. Les derniers chiffres donnés en mars 2017 par Chloe Corbyn, du service recherche de l'Assemblée, indiquent une progression constante : $62 \%$ de déchets recyclés contre $44 \%$ pour l'Angleterre ${ }^{19}$. Selon Rebecca Colley-Jones, spécialiste du recyclage et des ressources à l'Université de Bangor, de tels progrès sont à mettre sur le compte du cadre législatif imposé par l'Assemblée: "Le Gouvernement gallois est la seule administration dévolue qui a inscrit des objectifs de recyclage réglementaires dans sa législation jusqu'en $2025^{20} »$.

Malgré une couverture médiatique convenable, de tels succès n'occupent pas la une des journaux et ne contribuent guère à la visibilité du pays de Galles. Ils sont en fait éclipsés par les résultats mitigés obtenus en matière de réduction des émissions de gaz à effet de serre, ce que soulignait en octobre 2016 Jessica McQuade, de WWF Cymru :

Le Gouvernement n'a cessé d'exposer son ambition lors de conférences internationales et de tenir des propos justes. Après avoir subi beaucoup de pression de notre part, il a même mis en place une obligation réglementaire de réduire les émissions de gaz à effet de serre. Mais les statistiques sur la réduction de ces émissions et les preuves d'une action gouvernementale [...] racontent une tout autre histoire ${ }^{21}$.

21 En effet, les chiffres rendus publics par le National Atmosphere Emissions Inventory indiquent que le pays de Galles a réduit ses émissions de seulement $18 \%$ par rapport à 1990, alors que l'objectif établi dans le Environment Act 2016 est d'atteindre une baisse de $80 \%$ d'ici 2050. L'Écosse a quant à elle réduit ses émissions de $46 \%$. De telles différences s'expliquent notamment par la présence au pays de Galles de centrales au charbon et d'industries lourdes. Le pays de Galles était pourtant présent à la Conférence de Paris sur le climat, représenté par Carl Sargeant, Ministre des ressources naturelles, qui expliquait le 15 décembre 2015 :

Le Programme gouvernemental du Gouvernement gallois définit notre engagement à montrer la voie sur la question du réchauffement climatique et, ainsi, à contribuer à un accord international sur le réchauffement, le pays de Galles se positionnant comme un exemple pour d'autres états et régions plus petits. [...] J'ai aussi présenté le travail que nous menons ici au pays de Galles ${ }^{22}$. 
22 À l'occasion de la conférence de Paris, le pays de Galles devient l'un des premiers signataires de l'initiative RegionsAdapt, qui s'intéresse aux opérations menées à l'échelle supranationale pour appliquer les mesures adoptées à l'échelle internationale. Carl Sargeant fut également invité, tout comme une poignée de représentants de gouvernements nationaux et régionaux considérés comme leaders dans le domaine environnemental, à une rencontre privée avec Ban Ki-moon. Une marche est même organisée à Londres le 29 novembre 2015, au moment du lancement de la Conférence de Paris sur le climat, et Charlotte Church, chanteuse galloise, se produit à la fin de l'événement ${ }^{23}$. Seulement, Jessica McQuade dénonce le décalage entre théorie et pratique, la « dichotomie entre intention et mise en œuvre ${ }^{24} »$.

\section{Théorie et pratique}

La dichotomie entre intention et mise en œuvre, entre la théorie présentée dans les nombreuses publications de l'Assemblée et la politique finalement mise en pratique est une accusation habituelle à l'encontre des institutions galloises qui publient de nombreux documents et présentent de multiples projets sans réellement entrer dans le détail ${ }^{25}$. Ainsi les indicateurs permettant de mesurer le bien-être des Gallois et présentés dans le Well-being of Future Generations Act ne permettent pas réellement de prendre en compte le réchauffement climatique, pourtant l'un des défis majeurs du XXIe siècle. Cet écueil s'explique par les pouvoirs limités de l'Assemblée et sa volonté de promouvoir le bienêtre à l'échelle galloise et non planétaire, ce qui conduit les auteurs du texte à insister davantage sur le pilier social du développement durable, présent dans quatre objectifs sur six. Le gouvernement introduit ainsi en 2016 des réformes importantes du système éducatif, annonçant une révision des programmes à l'école primaire et secondaire, et une refonte du système de bourses dans l'enseignement supérieur. Ces mesures sont encore trop récentes pour que leur impact puisse être mesuré. Ces indicateurs n'apportent en outre aucune précision sur les moyens financiers ou les démarches innovantes dont les services publics auront besoin pour équilibrer besoins actuels et futurs, ce que souligne Chris Johnes dans son évaluation du projet de loi.: "Penser et planifier d'une façon qui permette d'équilibrer le présent et l'avenir assez lointain demandent de nouvelles mentalités et de nouvelles compétences qui seront évidemment plus naturelles chez certains que chez d'autres, que ce soit au niveau individuel ou institutionnel ${ }^{26} »$. Il regrette que le secteur privé ne soit pas concerné par la loi et insiste sur la nécessité de repenser le rôle que les acteurs non étatiques peuvent jouer dans l'application du principe de développement durable.

Quant au Environment Act, qui fixe des objectifs ambitieux pour la réduction des émissions de gaz à effet de serre au pays de Galles d'ici 2050, il ne les accompagne d'aucune obligation de produire rapidement des budgets et ne prévoit aucun objectif intermédiaire avant fin 2018-début 2019, soit à mi-mandat, ce que dénonce Jessica McQuade : «Par conséquent, nous courons le risque d'avoir un gouvernement menacé de ne pas donner de détails et de n'entreprendre aucune réduction substantielle des émissions durant une grande partie de son mandat ${ }^{27} "$.

De la même façon, ces deux lois entrent en totale contradiction avec un projet pourtant défendu depuis plusieurs années par l'Assemblée : le développement de la M4, autour de la ville de Newport, surnommée "the Black Route ${ }^{28}$. Lors du lancement de l'enquête publique le 28 février 2017, de nombreux spécialistes ont dénoncé ce projet, lourd de conséquences pour la biodiversité et générateur d'émissions de $\mathrm{CO}_{2}$, selon Rachel Sharp, 
directrice de Wildlife Trusts Wales, et sans aucune garantie de retombées économiques pour la région. Elle insiste sur ce décalage entre les engagements législatifs de l'Assemblée et ses projets :

Le Gouvernement gallois a récemment introduit une nouvelle législation pour lutter contre les menaces que représentent le réchauffement climatique, la perte de biodiversité et le manque d'écosystèmes résilients dans le Environment (Wales) Act 2016 et le Well-being of Future Generations (Wales) Act 2015. Les propositions faites par le gouvernement ne vont donc pas lui permettre de remplir ses propres engagements sur ce qu'il qualifie de législation 'révolutionnaire'29.

largement dominés par les discussions autour du Brexit et de ses conséquences pour le pays de Galles. Ainsi le 23 janvier 2017 Carwyn Jones et Leanne Wood, à la tête de Plaid Cymru, publiaient ensemble un livre blanc intitulé Securing Wales' Future, afin de préserver les intérêts gallois lors des négociations de sortie de l'UE, notamment: «le maintien des protections et valeurs sociales et environnementales que nous chérissons au pays de Galles, notamment les droits des travailleurs, une fois que celles-ci ne seront plus garanties par l'appartenance du Royaume-Uni à l'UE ${ }^{31}$ ». Dans le livre blanc, chapitre 8, les deux chefs de parti expliquaient :

Nous restons résolus à préserver les importantes protections sociales, environnementales et salariales qui ont été garanties par l'appartenance à l'UE. Elles ne doivent pas être fragilisées après la sortie de l'UE. [...] Il doit y avoir également un cadre spécifique, efficace et transparent à l'échelle britannique pour apporter un fondement juridique à la réglementation de sujets comme l'environnement, l'agriculture et la pêche qui sont largement gouvernés par le droit européen ${ }^{32}$.

Le pays de Galles est, d'autre part, en proie à une frénésie législative. Les institutions galloises travaillent en effet en étroite collaboration avec les instances européennes, en particulier en matière d'environnement, ce que soulignait Lesley Griffiths, Ministre de 
l'environnement et des affaires rurales, le 2 février 2017, lors de visites de terrain pour évoquer avec les agriculteurs d'une part, les organisations environnementales et le secteur agro-alimentaire, d'autre part, l'avenir de la filière post-Brexit :

Le portefeuille de l'environnement et des affaires rurales est, probablement plus que n'importe quel autre, grandement influencé par les financements et le droit européen. [...] Le retrait du Royaume-Uni de l'UE aura sans aucun doute un impact majeur sur les individus, les entreprises et les organisations représentés par mon portefeuille ${ }^{33}$.

De nombreuses régulations et directives environnementales émanent de l'Union européenne, ce que soulignaient Susan Baker et Katarina Eckeberg dans un article publié en janvier 2012: «L'Union européenne, de par son rôle de régulateur et par la formulation de projets stratégiques, comme la EU Sustainable Development Strategy, exerce une influence majeure sur la politique de développement durable. Les stratégies à l'échelle des états-nations sont conçues pour respecter les réglementations de l'UE ${ }^{34} »$. La sortie de l'UE fait craindre à l'Assemblée de perdre certaines de ses prérogatives ou le cadre imposé par plusieurs normes européennes lors de leur transfert de l'Union vers le Royaume-Uni, comme indiqué dans Securing Wales' Future, précédemment cité :

[L]e projet de loi ['Great Repeal Bill'] pourrait avoir un impact significatif, volontairement ou non, sur les compétences législatives de l'Assemblée nationale pour le pays de Galles, et le point central de notre politique est de faire en sorte que la sortie du Royaume-Uni de l'UE ne doit pas conduire à la récupération de pouvoirs dévolus par le Gouvernement britannique ${ }^{35}$.

C'est la raison pour laquelle deux membres de Plaid Cymru, Steffan Lewis AM, porte-parole du parti nationaliste pour le Brexit, et Simon Thomas AM, porte-parole pour les affaires rurales, ont proposé un Welsh European Union Continuity Bill pour intégrer dans le droit gallois toutes les directives européennes existantes, respectées pour l'instant du fait de l'appartenance à l'Union. Selon Steffan Lewis :

Le pays de Galles a besoin d'un EU Continuity Bill pour inscrire toutes les réglementations existantes dans le droit gallois. Cela garantira que les normes que nous apprécions, telles que les protections environnementales, les normes alimentaires, et les droits que nous considérons aujourd'hui comme acquis en tant que citoyens européens, s'appliquent toujours au pays de Galles après le Brexit. Cela empêchera aussi que le EU Repeal Bill des Conservateurs ne devienne une prise de pouvoir de Westminster sur des domaines déjà dévolus ${ }^{36}$.

33 Ainsi, l'Assemblée essaye d'adopter un grand nombre de mesures dans les domaines dévolus, notamment l'environnement, afin de les intégrer dans le droit avant la sortie de l'UE, et, aussi, avant le passage au modèle des domaines réservés, introduit par le Wales Act 2017. À partir d'avril 2018, le pays de Galles sera doté du même cadre constitutionnel que l'Écosse, c'est-à-dire que l'Assemblée pourra légiférer dans tous les domaines, sauf ceux mentionnés dans le Wales Act 2017, dits réservés, comme la sécurité nationale.

\section{Conclusion}

En conclusion, le pays de Galles, depuis la mise en place de ses institutions en 1998, cherche à se présenter comme un modèle à suivre, notamment grâce à sa politique environnementale dont le fer de lance est le développement durable, au cœur de sa politique, ainsi que de celle des organismes publics gallois depuis l'adoption de deux lois majeures, le Well-being of Future Generations Act et le Environment (Wales) Act. Même s'il est 
encore tôt pour dresser un bilan de ces mesures, elles ne semblent pas pour l'instant avoir permis au pays de Galles de gagner en visibilité, d'autant plus que le gouvernement n'a pas encore réellement mis en pratique les engagements théoriques pris dans ces documents. Il est également difficile, pour l'instant, de mesurer leur impact sur le quotidien des Gallois. Enfin, le Brexit place l'Assemblée dans une situation paradoxale: elle doit s'empresser d'incorporer dans la législation galloise des directives environnementales européennes de peur que le gouvernement britannique ne les abandonne, tout en tentant de négocier avec une Premier ministre affaiblie, encore davantage depuis les élections anticipées du 8 juin 2016. L'Assemblée adopte en urgence le Wales EU Continuity Act le 21 mars 2018, afin de préserver le cadre constitutionnel de la dévolution. Ayant obtenu un compromis du gouvernement britannique, qui accepte d'amender le $E U$ (Withdrawal) Bill, de telle sorte que la liste des prérogatives conservées par Westminster sera définie conjointement par le gouvernement central et les administrations dévolues et que celles-ci lui seront confiées pour une période maximale de cinq ans, le gouvernement gallois accepte le projet de loi. Il est soumis au vote de l'Assemblée le 15 mai 2018 et approuvé en séance plénière. Le First Minister, Carwyn Jones, doit poursuivre les négociations sur la question du marché unique et de l'espace douanier, tentant de préserver les intérêts gallois, tout en respectant le vote des électeurs gallois qui se sont prononcés en majorité en faveur du Brexit.

\section{BIBLIOGRAPHIE}

CARDIFF UNIVERSITY, “Mapping future emissions in Wales”, 02-03-2017, https://www.cardiff.ac.uk/ news/view/619289-mapping-future-emissions-in-wales, consulté en mai 2017.

CORBYN Chloe, "Wales leads the way on recycling", 10-03-2017, Cardiff, Welsh Assembly, https:// seneddresearch.blog/2017/03/10/wales-leads-the-way-on-recycling/, consulté en mai 2017. DEVINE Darren, "Why does the Paris climate change conference matter?", Cardiff, WalesOnline, 30-11-2015, http://www.walesonline.co.uk/news/wales-news/paris-climate-change-conferencematter-10524775, consulté en juin 2017.

GREAT BRITAIN, GOWA 1998, Londres, HMSO, c. 38, 1998.

GREAT BRITAIN, GOWA 2006, Londres, HMSO, c. 32, 2006.

GRIFFITHS Lesley, “Lesley Griffiths to hear views on Securing Wales' future”, Cardiff, Welsh Government, 02-02-2017, http://gov.wales/newsroom/environmentandcountryside/2017/170202lesley-griffiths-to-hear-views-on-securing-wales-future/?lang=en, consulté en avril 2017. HOwE Sophie, “Yet Another Road”, Cardiff, IWA, clickonwales, 24-02-2017, http://www.iwa.wales/ click/2017/02/yet-another-road/, consulté en avril 2017.

JOHNES Chris, Understanding the Wellbeing of Future Generations Bill, an Egino Analysis, Cardiff, Egino CIC, février 2013. 
KEEP WALES TIDY, "Well-being of Future Generations Act anniversary", Cardiff, Keep Wales Tidy, 03-04-2017, https://www.keepwalestidy.cymru/news/well-being-of-future-generations-actanniversary, consulté en avril 2017.

MCQUADE Jessica, "Wales is slipping back on climate change action - can this government close the 'ambition gap' ?", IWA, 13-10-2016, www.clickonwales, ??

MCQUADE Jessica, "Message in a Bottle”, Cardiff, Institute of Welsh Affairs, clickonwales, 17-03-2017, http://www.iwa.wales/click/2017/03/message-in-a-bottle/, consulté en avril 2017.

MESSENGER Steffan, “Wales 'could become Europe's top recycling nation”', 28-10-2016, http:// www.bbc.com/news/uk-wales-37787961, consulté en avril 2017.

MILLER Auriol, “Carl Sargeant's record”, IWA, clickonwales, 15-12-2017, http://www.iwa.wales/ click/2017/12/carl-sargeants-record/, consulté en décembre 2017.

MORRIS Steven, "Plastic bag use down 70\% in Wales since charges began", 04-09-2015, The Guardian , https://www.theguardian.com/environment/2015/sep/04/plastic-bag-use-down-70-walessince-charges-began, consulté en mai 2017.

NICHOLl Anna et OSMOND John, Wales' Central Organising Principle, Cardiff, Institute of Welsh Affairs, 27-01-2012.

RAISIN Claire, "Wales and climate change : let's be part of the solution", IWA, clickonwales, 06-10-2016, http://www.iwa.wales/click/2016/10/wales-climate-change-lets-part-solution/, consulté en avril 2017.

SARGEANT Carl, "Written statement - United Nations Framework Convention on Climate Change (UNFCC) - Paris”, Cardiff, Welsh Government, 15-12-2015, http://gov.wales/about/cabinet/ cabinetstatements/previous-administration/2015/climatechangeparis/?lang=en, consulté en avril 2017.

SHARP Rachel, “A David and Goliath battle”, Cardiff, IWA, clickonwales, 28-02-2017, http:// www.iwa.wales/click/2017/02/david-goliath-battle/, consulté en avril 2017.

WELSH ASSEMBLY, One Wales, One Planet, Sustainable Development Scheme, Cardiff, Welsh Assembly, 2009. WELSH ASSEMBLY, Well-being of Future Generations Act, Cardiff, Welsh Assembly, 2015.

WELSH ASSEMBLY, Environment (Wales) Act, Cardiff, Welsh Assembly, 2016.

WELSH GOVERNMENT et CYNNAL CYMRU-SUSTAIN WALES, The Wales We Want, 08-08-2016, https:// www.thewaleswewant.co.uk.

WELSH GOVERNMENT, "Securing Wales' Future: First Minister Carwyn Jones and leader of Plaid Cymru Leanne Wood set out Wales' post-referendum plan”, Cardiff, Welsh Government, 23-01-2017, http://gov.wales/newsroom/firstminister/2017/170123-brexit-plan/?lang=en, consulté en juin 2017.

WELSH GOVERNMENT, Securing Wales' Future, Transition from the European Union to a new relationship with Europe, Cardiff, Welsh Government, 2017.

WILliams Phil, “A Green Wales”, Agenda, Cardiff, IWA, été 2003. 


\section{NOTES}

1. "The Assembly shall make a scheme setting out how it proposes, in the exercise of its function, to promote sustainable development", GOWA 1998 (Londres, HMSO, 1998), c. 38 Part 5, section 121.

2. "In a small forgotten country like Wales we do have an inferiority complex and we often boast without foundation that our role is to lead the world. A poet friend of mine thinks it all springs from the memory of Magnus Maximus who set out from Wales and ended up Roman Emperor north of the Alps. But who knows: if we have any chance of making our mark as a small nation it must be as a Green nation and as a nation that takes its commitment to sustainable development very seriously", Phil Williams, présentation donnée en 2002 lors d'un colloque intitulé "Regio nalism and Sustainable Development» organisé à Bad Urach, Allemagne, en juin 2002 et reproduite dans Agenda, Cardiff, IWA, été 2003, pp. 2-5.

3. "The Wales Future Generations Act captures the spirit and essence of two decades of United Nations work in the area of sustainable development and serves as a model for other regions and countries. [...] We hope that what Wales is doing today the world will do tomorrow", Nikhil Seth, op. cit.

4. "We will legislate to embed sustainable development as the central organising principle in all our actions across government and all public bodies, by bringing forward a sustainable development Bill. The approach will set Wales apart as a sustainable nation, leading from the front", Carwyn Jones, cité dans Chris Johnes, Understanding the Wellbeing of Future Generations Bill, an Egino Analysis (Cardiff, Egino CIC, 2013), p. 4.

5. C'est la définition donnée dans le rapport Brundtland, ou Our Common Future, publié en 1987 par la Commission mondiale sur l'environnement et le développement de l'ONU, présidée par Gro Harlem Brundtland. C'est dans ce rapport que l'expression « développement durable » est utilisée pour la première fois avec la définition suivante : "Le développement durable est un mode de développement qui répond aux besoins des générations présentes sans compromettre la capacité des générations futures de répondre aux leurs ».

6. "The Well-being of Future Generations Bill strengthens the existing governance arrangements in named public sector organisations for improving the well-being of Wales. The Bill ensures that the needs of the present are met without compromising the ability of future generations to meet their own needs. This will be provided for by securing the sustainable development principle in law", Jeff Cuthbert, cité dans Chris Johnes, op. cit., p. 5. La définition dite Brundtland est celle couramment retenue pour définir le développement durable. Elle est tirée du rapport de la Commission mondiale sur l'environnement et le développement de l'Organisation des Nations Unies, dit Rapport Brundtland, du nom de sa présidente, où cette expression est apparue pour la première fois en 1987.

7. "When Carl came into post, he was the fourth Minister who had worked on the Well-being of Future Generations Act. It was not popular and was not effective. Carl was initially unconvinced about how it would help people. But he listened and responded to our requests for amendments, and once he got behind the legislation he was very effective at getting it through. The result was a truly radical law to ensure that sustainable development is at the heart of Government and public services, which has won global recognition", Anne Meikle, citée dans Auriol Miller, "Carl Sargeant's record", IWA, clickonwales, 15-12-2017, http://www.iwa.wales/click/2017/12/carlsargeants-record/, consulté en décembre 2017.

8. "This Act is about improving the social, economic, environmental and cultural well-being of Wales", The Wales We Want, www.gov.wales, 08-08-2016, consulté en avril 2017. 
9. "The better news is that we are now in a stronger position than a few years ago to face this challenge [a low carbon Wales] thanks to the Welsh Government. We have in place brand new legislation to help change the way decisions are made. The Environment Act has introduced statutory targets for Welsh Ministers to reduce emissions and provides a clear plan for implementation. The Wellbeing of Future Generations Act requires climate considerations to be integrated into decisions made by public bodies", Jessica McQuade, "Message in a Bottle", IWA, clickonwales, 17-03-2017, www.iwa.wales, consulté en avril 2017.

10. L'avenir des aciéries Tata employant 4000 personnes à Port Talbot a été au cœur des débats lors des dernières élections galloises de mai 2016, tous les chefs de parti s'étant engagés à résoudre la "crise de l'acier », provoquée par l'annonce de Tata Steel de fermer tous ses sites gallois.

11. Voir à ce sujet "Mapping future emissions in Wales", 02-03-2017, www.cardiff.ac.uk, consulté en mai 2017.

12. Cinq pence par sac depuis le $1^{\mathrm{er}}$ octobre 2011, versés à des œuvres caritatives, et il était estimé en septembre 2015 que l'utilisation de ces sacs avait chuté de $70 \%$ depuis, voir Steven Morris, "Plastic bag use down 70\% in Wales since charges began", 04-09-2015, The Guardian, www.theguardian.com, consulté en janvier 2017.

13. Ce titre est attribué par un panel d'experts britanniques et européens du commerce équitable.

14. Claire Raisin, "Wales and climate change : let's be part of the solution", IWA, clickonwales, 06-10-2016, http://www.iwa.wales/click/2016/10/wales-climate-change-lets-part-solution/, consulté en avril 2017.

15. "Wales does have the potential to influence other countries' policies, showcasing what can be done when fighting climate change is prioritised. Wales could absolutely make a name for itself on the world stage, and for all the right reasons", Loc. cit.

16. "The anniversary of the Well-being and Future Generations Act is an opportunity to highlight the progress made in the past year. Wales continues to lead the way with sustainability legislation recognised by the UN as 'a model for other regions and countries", Lesley Griffiths, citée dans «Well-being of Future Generations Act anniversary », 03-04-2017, www.keepwalestidy.cymru, consulté en avril 2017.

17. Voir Steffan Messenger, correspondant de BBC Wales pour l'environnement, "Wales 'could become Europe's top recycling nation”,' 28-10-2016, http://www.bbc.com/news/ukwales-37787961, consulté en avril 2017.

18. Ibid.

19. Chloe Corbyn, "Wales leads the way on recycling", 10-03-2017, https:// seneddresearch.blog/2017/03/10/wales-leads-the-way-on-recycling/, consulté en avril 2017.

20. "The Welsh Government is the only devolved administration to have put statutory recycling targets in legislation up to 2025", citée dans Steffan Messenger, op. cit.

21. "The Government has constantly set its ambition at international conferences and said the right things. After a lot of pressure from our sector, it even created a statutory obligation to reduce emissions. But the statistics on emission reduction and evidence of government action [...] tell a very different story", Jessica McQuade, "Wales is slipping back on climate change action can this government close the 'ambition gap' ?", IWA, 13-10-2016, www.clickonwales, consulté en avril 2017.

22. "The Welsh Government's Programme for Government sets out our commitment to leadership on climate change and in doing so to contributing to a global deal on climate change, with Wales acting as an exemplar to other smaller states and regions. [...] I also showcased the work we are undertaking here in Wales", Carl Sargeant, «Written statement - United Nations Framework Convention on Climate Change (UNFCC) - Paris", 15-12-2015, http://gov.wales/ 
about/cabinet/cabinetstatements/previous-administration/2015/climatechangeparis/?lang=en, consulté en avril 2017.

23. Darren Devine, "Why does the Paris climate change conference matter ?", 30-11-2015, http:// www.walesonline.co.uk/news/wales-news/paris-climate-change-conference-matter-10524775, consulté en juin 2017.

24. "[a] dichotomy between intention and delivery", Jessica McQuade, op. cit.

25. Déjà en janvier 2012, Peter Davies soulignait le fossé entre l'adoption du principe de développement durable, tel qu'imposé par le GOWA 1998 et réaffirmé par le GOWA 2006, et la mise en application de celui-ci: "Il [le devoir du Gouvernement gallois de promouvoir le développement durable] est aussi reconnu dans le monde comme un exemple à suivre de l'intégration du principe de développement durable dans les structures gouvernementales. [...] Néanmoins, et on peut le comprendre, subsiste un fossé significatif entre l'adoption du principe et son application comme mode décisionnel à travers le gouvernement " ("It [the Welsh Government's duty to promote sustainable development] is also recognised internationally as being a leading example of the integration of sustainable development within government structures. [...] Nonetheless, and understandably, there remains a significant gap between the adoption of the principle and its implementation as a way of decision-making across government”, Peter Davies, “A crucial step in Wales' devolution journey”, in Anna Nicholl \& John Osmond, Wales' Central Organising Principle, Cardiff, IWA, 2012, p. 45-46.

26. "To think and plan in ways that balance the present and the quite distant future requires new mindsets and new skills which will clearly come more easily to some than to others, both individually and institutionally", Chris Johnes, op. cit., p. 9. Il qualifie cette difficile mise en pratique de la loi ainsi : "the Delivery Challenge".

27. "As a consequence, we run the risk of having a government in danger of providing no detail and undertaking no substantial emission reduction during most of its term of office", Jessica McQuade, op. cit.

28. L'idée de soulager la M4 autour de Newport en construisant une deuxième portion d'autoroute entre Magor et Castleton fut introduite pour la première fois par le Welsh office en 1991. Elle fut réintroduite par l'Assemblée en 2006, puis abandonnée du fait du coût des travaux estimés à un milliard de livres sterling, et relancée en 2011 après l'annonce du gouvernement britannique d'apporter une contribution financière. Le projet final, la «Black Route ", autoroute à six voies, a été retenu contre la « Red Route » et la « Purple Route ».

29. "The Welsh Government have recently introduced new legislation to tackle threats posed by climate change, the loss of biodiversity and the lack of resilient ecosystems in the Environment (Wales) Act 2016 and the Well-being of Future Generations (Wales) Act 2015. The government proposals will therefore fail to meet their own commitments on what they call 'ground-breaking' legislation”, Rachel Sharp, “A David and Goliath battle”, IWA, clickonwales, 28-02-2017, http:// www.iwa.wales/click/2017/02/david-goliath-battle/, consulté en avril 2017.

30. "Building a new road is not in the interests of future generations. [...] As the Future Generations Commissioner for Wales it is my role to ensure that public bodies are fulfilling their duties in relation to the Well-being of Future Generations Act. The things we do now to prepare for this must ensure we're thinking to 2036 and beyond, rather than falling back on a 1996 solution of building yet another road", Sophie Howe, "Yet Another Road", IWA, clickonwales, 24-02-2017, http://www.iwa.wales/click/2017/02/yet-another-road/, consulté en avril 2017.

31. "[m]aintaining the social and environmental protections and values that we prize in Wales, in particular workers' rights, once these are no longer guaranteed through the UK's membership of the EU", "Securing Wales' Future: First Minister Carwyn Jones and leader of Plaid Cymru Leanne Wood set out Wales' post-referendum plan", 23-01-2017, http://gov.wales/newsroom/ firstminister/2017/170123-brexit-plan/?lang=en, consulté en juin 2017. 
32. "We remain committed to the important social, environmental and employment protections which have been secured through EU membership. They must not be undermined after leaving the EU. [...] There must also be a proper, effective and transparent UK framework to provide legal underpinning for effective regulation of issues such as environment, agriculture and fisheries which are heavily governed by EU law", Welsh Government, Securing Wales' Future, Transition from the European Union to a new relationship with Europe, Welsh Government, Cardiff, 2017, p. 32.

33. "The Environment and Rural Affairs portfolio is, probably more than any other, hugely impacted by EU funding and legislation. [...] The UK's withdrawal from the EU will undoubtedly have a major impact on individuals, businesses and organisations represented by my portfolio", Lesley Griffiths, "Lesley Griffiths to hear views on Securing Wales' future", 02-02-2017, http:// gov.wales/newsroom/environmentandcountryside/2017/170202-lesley-griffiths-to-hear-viewson-securing-wales-future/?lang=en, consulté en avril 2017.

34. "In its role as regulator and in the formulation of strategic plans, for example the EU Sustainable Development Strategy, the European Union has a major influence on sustainable development policy. Strategies at the nation-state level are devised to comply with EU requirements", Susan Baker \& Katarina Eckerberg, "Sustainable Development within the EU", in Anna Nicholl \& John Osmond, Wales' Central Organising Principle, op. cit., p. 63.

35. "[T]he Bill ['Great Repeal Bill'] may significantly impact, intentionally or not, on the legislative competence of the National Assembly for Wales, and our core standing policy is that the UK exit from the EU must not result in devolved powers being clawed back to the UK Government", Ibid., p. 28.

36. "Wales needs an EU Continuity Bill to enshrine all existing regulations into Welsh law. This will ensure that the standards we value, like environmental protections, food standards, and the rights we have come to take for granted as EU citizens, continue to apply to Wales after Brexit. It will also stop the Conservatives' EU Repeal Bill turning into a Westminster power-grab over already devolved matters", Site Plaid Cymru, www2.partyof.wales, consulté en janvier 2017.

\section{RÉSUMÉS}

Le Government of Wales Act 1998, qui a permis la mise en place d'une assemblée dévolue au pays de Galles dotée dans un premier temps de pouvoirs législatifs secondaires, faisait de la Principauté la première nation européenne à inclure le principe de développement durable dans le texte fondateur de ses institutions. Selon Phil Williams, AM (Assembly Member) pour Plaid Cymru, le parti nationaliste gallois, une telle décision devait faire du pays de Galles un modèle environnemental et lui apporter une grande visibilité. Pourtant, au cours de ses premières années d'existence, le bilan de l'Assemblée reste mitigé, notamment en raison des pouvoirs limités dont elle jouit. Elle obtient en 2011 le statut de parlement et bénéficie désormais de pouvoirs législatifs primaires dans les domaines dévolus, ce qui lui permet d'adopter deux lois majeures pour le développement durable : le Well-Being of Future Generations Act en 2015 et le Environment (Wales) Act l'année suivante, lois qui placent le pays de Galles sur le devant de la scène internationale. Cet article se propose de présenter ces deux lois majeures adoptées par l'Assemblée, afin de dresser le bilan de son action, notamment le gain de visibilité du pays de Galles, les conflits entre théorie et pratique, enfin la question du Brexit qui plonge les institutions galloises dans un paradoxe législatif. 
The Government of Wales Act 1998, which set up an assembly for Wales with, originally, secondary legislative powers, made Wales the first country in Europe to include sustainable development in its founding document. Phil Williams, Plaid Cymru AM, considered that such a decision would allow Wales to become an environmental model and to gain in visibility. Yet, during its first years, the Assembly, flawed from the outset by its limited powers, was not seen as very successful. In 2011 the Assembly was granted full law-making powers in all devolved areas, allowing the voting of two major environmental acts - the Wellbeing of Future Generations Act in 2015 and the Environment (Wales) Act the following year - putting Wales in the limelight. This article aims at analysing these two acts voted by the Welsh Assembly, assessing their implementation and indicating whether Wales has gained visibility on this question despite the conflicts between its theory and practice. Finally the perspective of Brexit, which puts the country in a paradoxical legislative position, will be examined.

\section{INDEX}

Mots-clés : Pays de Galles, développement durable, environnement

Keywords : Wales, sustainable development, environment

\section{AUTEUR}

\section{STÉPHANIE BORY}

Institut d'Etudes Transtextuelles et Transculturelles (IETT), EA 4186, Université Jean Moulin Lyon 3 